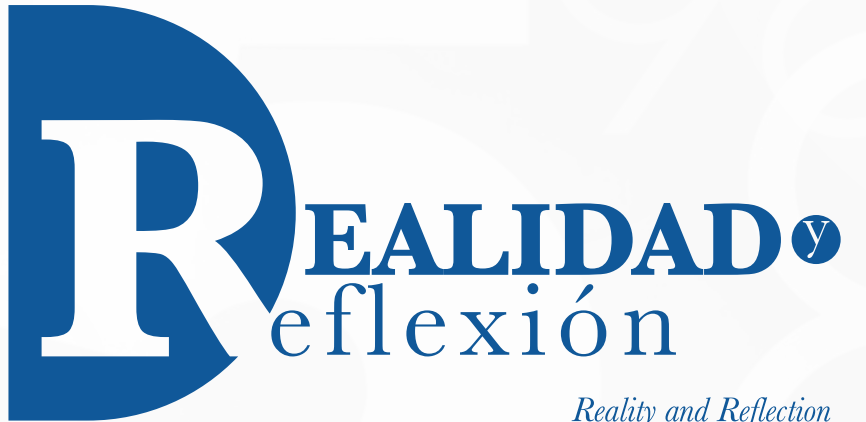

ISSN 1992-6510

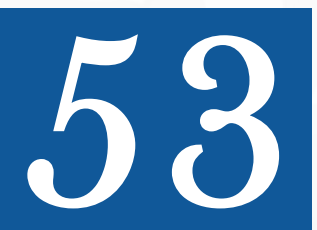

Año 21, N 53, San Salvador, El Salvador, Centroamérica. Revista Semestral Enero-Junio 2021

YEAR 21, N53, SAN SALVADOR, EL SALVADOR, CENTRAL AMERICA. SEMESTRAL JOURNAL JANUARY-JUNE 2021

\title{
El desarrollo del pensamiento algebraico en estudiantes al inicio de la escolarización
}

\section{O desenvolvimento do pensamento algébrico de estudantes em inicio de escolarização}

\section{The development of algebraic thinking of students at the beginning of schooling} Kátia Gabriela Moreira
Universidade São Francisco (USF) ktiagabriela@hotmail.com

Adair Mendes Nacarato Universidade São Francisco (USF) ada.nacarato@gmail.com

Recibido: 29 de junio de 2020 Aceptado: 11 de septiembre de 2020 DOI: $10.5377 /$ ryr.v53i53.10894 


\section{RESUMEN}

El presente artículo se centra en el movimiento de producir de significados relacionados al pensamiento algebraico. La investigación fue realizada a lo largo del año lectivo 2017 en una clase de primer año de Educación Básica ${ }^{\mathrm{i}}$ que comprende niños de seis años de edad - compuesta por 26 alumnos de una escuela municipal del interior del estado de São Paulo, Brasil. Para la documentación de la investigación, las clases de la profesora-investigadora fueron grabadas en video sirviendo de apoyo para la escritura de narrativas pedagógicas. En estas narrativas se realiza el análisis microgenético dirigido hacia los indicios que permiten la interpretación de los episodios observados, permitiendo la aproximación a los procesos referentes al desarrollo del pensamiento algebraico de los alumnos. El análisis muestra que los alumnos del primer año de educación básica comprometidos en una cultura social de salón de clase de matemática enmarcada en la problematización, y en la cual son protagonistas de su propio proceso de aprendizaje, son capaces de generalizar el patrón de la secuencia por medio del lenguaje materno.

Palabras clave: Desarrollo del pensamiento algebraico, años iniciales, investigación de la práctica de enseñanza propia.

\section{RESUMO}

O presente artigo centra-se no movimento de produção de significados relacionados ao Pensamento Algébrico. A investigação foi realizada ao longo do ano letivo de $2017 \mathrm{em}$ uma sala de aula de um primeiro ano do Ensino Fundamental — que atende crianças de 6 anos de idade - composta por 26 alunos de uma escola municipal no interior do estado de São Paulo, Brasil. Para a documentação da pesquisa, as aulas da professora-pesquisadora foram videogravadas e serviram de apoio para a escrita de narrativas pedagógicas. Nestas, realiza-se a análise microgenética orientada para os indícios que permitem a interpretação dos episódios observados, possibilitando a aproximação com os processos de desenvolvimento do Pensamento Algébrico dos alunos. A análise aponta indícios de que os estudantes do primeiro ano do Ensino Fundamental, engajados numa cultura social de sala de aula de Matemática, pautada na problematização, na qual são protagonistas do próprio processo de aprendizagem, são capazes de generalizar o padrão da sequência por meio da linguagem materna.

Palavras-chave: desenvolvimento do Pensamento Algébrico, anos iniciais, pesquisa da própria prática.

\section{ABSTRACT}

This article focuses on the motion of production of meanings related to algebraic thinking. The investigation was carried out throughout the academic year of 2017 in a classroom of a first year of elementary school - which serves children of six years of age - composed of 26 students from a municipal school in the interior of the state of São Paulo, Brazil. The teacher-researcher's classes were videotaped as a means of research documentation and served as support for the writing of pedagogical narratives. A microgenetic analysis of the narratives is 
carried out oriented to the indications that allow the interpretation of the episodes observed, enabling the approximation of the processes related to the development of students' algebraic thinking. The analysis of the pedagogical narrative points to evidence that the students of the first year of elementary school, engaged in a social culture of the mathematics classroom based on problematization and in which they are protagonists of their own learning process, are able to generalize the pattern sequence through their maternal language.

Keywords: Development of algebraic thinking, early years, research of own teaching practice. 


\section{Introducción}

El presente artículo se refiere a una parte de una investigación más amplia en el ámbito de doctorado (desarrollado por la primera autora y orientado por la segunda), que analiza narrativamente la práctica de la profesora-investigadora que buscó desarrollar el pensamiento algebraico (PA) de los alumnos de primer año (6 años de edad) de educación básica. En la sección aquí presentada, centramos nuestra mirada en la producción de significados relacionados al PA a partir de una tarea que involucra la percepción y la generalización del patrón de una secuencia recursiva.

La investigación sobre el PA en los primeros años es reciente en Brasil. Las discusiones oficiales frente a este campo surgen en el 2012 a partir de la publicación de un documento realizado por el Ministerio de Educación (2012), en el cual, el área de Matemática aparece organizada en cinco ejes: Números y Operaciones, Pensamiento Algebraico, Espacio y Forma, Magnitudes y Medidas y Tratamiento de la Información. Desde esa época, los documentos curriculares pasan a contemplar el eje del PA desde el inicio del proceso de escolarización de los alumnos -hasta entonces, la enseñanza del álgebra era introducida en el currículo a partir del $7^{\circ}$ año, que comprende alumnos de 12 a 13 años de edad-. La Base Nacional Común Curricular - BNCC, cuya versión fue aprobada en 2017, es el documento curricular de ámbito nacional publicado más recientemente en Brasil. Después de algunas modificaciones a lo largo del proceso de elaboración, el eje Pensamiento Algebraico - presente en el documento de 2012 - es denominado como Álgebra.

Aunque en Brasil tales discusiones están en fase embrionaria, las investigaciones en países que desde hace tiempo discuten cuestiones referentes al trabajo con el PA de alumnos al inicio del proceso de escolarización revelan resultados favorables. Tales resultados se destacan en las investigaciones de Blanton y Kaput (2005), Carraher et al. (2008); Vale (2006); Mason (2007); Cyrino y Oliveira (2011); Mestre (2014); Radford (2008, 2012, 2013, 2014).

El artículo está organizado en cuatro apartados: algunas reflexiones teóricas sobre el desarrollo del pensamiento algebraico desde la perspectiva histórico-cultural; presentación de la metodología; la narrativa pedagógica de la profesora-investigadora; análisis de la narrativa de la profesora-investigadora.

\section{El desarrollo del pensamiento algebraico en estudiantes al inicio de la escolarización desde la perspectiva histórico-cultural}

En las discusiones de los autores, aunque con algunas variaciones en sus concepciones, existe consenso acerca de la potencialidad y de la necesidad de introducir el trabajo de desarrollo del PA desde los primeros años de la Enseñanza Básica, priorizando la construcción de los conceptos algebraicos como un proceso que se desdobla en un trabajo más amplio que incluye las propiedades de los números y de las operaciones, las igualdades numéricas, el cambio, los patrones y las relaciones entre cantidades y, 
que, aunque no introduzca necesariamente las notaciones algebraicas convencionales, permite el uso del lenguaje materno y de otras representaciones para expresar ideas algebraicas.

Si, por un lado, las investigaciones señalan la potencialidad y la necesidad de trabajar con el desarrollo del pensamiento algebraico desde el inicio del proceso de escolarización, por otra parte, aún existe una gran laguna en las investigaciones enfocadas a las implicaciones pedagógicas y los caminos posibles para realizar este trabajo en el salón de clase.

A partir de las divergencias en los documentos nacionales entre el uso de los términos Pensamiento Algebraico (BRASIL, 2012) y Álgebra (BRASIL, 2017), y más aún cuando en los textos teóricos encontramos la denominación "raciocinio algebraico", percibimos la necesidad de profundización teórica en búsqueda de una distinción entre tales términos. Sin embargo, en la propia literatura existen divergencias - lo que torna la distinción entre los términos una acción aún más difícil, dado que no existe consenso entre los autores. No obstante, varios estudios contienen importantes elementos que permiten caracterizar el PA.

Squalli (2000) señala la necesidad de distinguir entre Álgebra y PA, entendiendo el Álgebra como "un tipo de actividad matemática" y el pensamiento algebraico como "un conjunto de habilidades intelectuales que interviene en esas actividades" ( p. 277) que está constituido por tres componentes que comprenden (1) la construcción e interpretación de modelos algebraicos; (2) la manipulación de expresiones algebraicas a partir de reglas preestablecidas y (3) la elaboración y aplicación de estructuras y procedimientos algebraicos. E1 PA está constituido por las habilidades que posibilitan el pensar analíticamente sobre los componentes del Álgebra. A partir del PA, los alumnos generalizan y abstraen relaciones, reglas y estructuras, y manipulan el lenguaje algebraico. Así, mientras que el Álgebra puede ser concebida como un tipo particular de actividad Matemática, el PA es un conjunto de habilidades intelectuales necesarias al Álgebra. El Álgebra y el PA son presentados por el autor como conceptos complementarios e indisociables (Squalli, 2000).

De modo general, las autoras evidencian que la introducción del álgebra desde el inicio de la escolarización debe ser comprendida como el desarrollo de un modo de pensar que antecede al uso del lenguaje algebraico. Así, amparadas por los estudios teóricos, asumimos el uso de la expresión "pensamiento algebraico" dado que entendemos la relación dialéctica entre el pensamiento y el lenguaje, siendo coherentes con la perspectiva histórico-cultural en la cual está enmarcada la práctica pedagógica e investigativa del presente estudio. A partir de una revisión de literatura, Cyrino y Oliveira (2011) entienden el término Pensamiento Algebraico como "un modo de describir significados atribuidos a los objetos del álgebra, a las relaciones existentes entre ellos, a la modelación, y a la resolución de problemas en el contexto de la generalización de estos objetos" (p. 103). Otros autores del campo de la educación matemática discuten el PA partiendo de la perspectiva histórico-cultural, como ocurre con Radford (2012, 2013, 2014) y Mason (2007). 
Radford (2012), enmarcándose en las relaciones entre pensamiento y lenguaje discutidas por Vigotski, considera que el desarrollo del pensamiento requiere que se consideren:

Los varios componentes que lo intervienen (por ejemplo, percepción, gestos, habla, artefactos y símbolos). También es imperativo investigar la manera en la cual cada uno de estos componentes significa y se transforma a medida que nuevos complejos de significado surgen y evolucionan. (p. 121)

El desarrollo de la percepción es de la misma naturaleza que la del gesto o de la actividad simbólica, y estos se desarrollan de manera articulada. El pensamiento puede ser considerado "una práctica social materializada en el cuerpo (como acciones kinestésicas, gestos, percepción, visualización), en el uso de signos (símbolos matemáticos, gráficos, palabras escritas y habladas) y artefactos de diferentes tipos (reglas, calculadoras, etc.)" (Radford, 2013, p. 120). Para él, por lo tanto, el pensamiento es una unidad sistémica que incluye múltiples lenguajes y formas cultural e históricamente constituídas y, que por medio de mediaciones semióticas, puede ser desarrollado en el salón de clase. El reto consiste en investigar cómo esos diferentes componentes (percepción, gestos, habla, artefactos y símbolos) son transformados en procesos de significación y a la manera en la cual estos elementos se interrelacionan, se organizan y reorganizan en el desarrollo del pensamiento algebraico desde los primeros años. Como no se trata de un proceso innato, "es necesario considerar las condiciones contextuales que producen nuevas formas de pensamiento posible" (Radford, 2012, p. 121).

Apoyándose también en las relaciones entre pensamiento y lenguaje, Mason (2007) opina que la generalización es "el corazón de la Matemática", revelando así la importancia del proceso de generalización en el aprendizaje de la Matemática. El autor concibe la relación entre la palabra y el PA dado que la palabra es fundamental para el proceso de generalización. El autor incluso sostiene que los alumnos, ya desde el inicio del proceso de escolarización, son capaces de generalizar a partir de casos particulares, una vez que la generalización se presenta como una herramienta que el ser humano usa para darle sentido al mundo que le rodea. Sin embargo, hay que considerar la necesidad de que los alumnos estén inmersos en contextos que posibiliten la construcción de significados para los procesos de generalización; es decir, contextos en los que puedan expresar sus ideas, fortaleciendo y ampliando esta capacidad de generalización.

Los niños que se involucran en el proceso de generalización por medio de la palabra y de su significado están inmersos - todo el tiempo - en contextos de búsqueda de lo particular en lo general y de lo general en lo particular. Para Mason (2007), los seres humanos generan sentidos para sus experiencias y usan sus capacidades para recolectar, clasificar e, incluso, rechazar sentidos, sean físicos o imaginarios. De esta forma, la imaginación está significada en el proceso de desarrollo humano, lo que permitirá que las imágenes, los diagramas o los símbolos actúen como mediadores del proceso de aprendizaje y desarrollo de los alumnos. Sin embargo, esta capacidad de imaginación debe ser estimulada por medio 
de los diferentes contextos, particularmente el del salón de clase, haciendo posibles la expresión de regularidades y el establecimiento de relaciones (Mason, 2007).

La generalización se presenta como un aspecto fundamental de la enseñanza, particularmente en los años iniciales. Sin embargo, uno de los mayores retos de la enseñanza es la construcción y la ampliación de estos conceptos matemáticos de manera significativa para que los alumnos sean capaces -partiendo de esta construcción - de comunicar, representar y establecer conexiones matemáticas. Una forma de desarrollar la capacidad de generalización es sensibilizar a los niños para la distinción entre "mirar hacia y mirar a través de”, conjugándose esta última como la capacidad de ver la generalización a partir de lo particular.

Adoptamos en este trabajo la definición de Blanton y Kaput (2005), que caracterizan el PA como un "proceso en el que los alumnos generalizan ideas matemáticas a partir de un conjunto de ejemplos particulares, establecen esta generalización a través de la argumentación, y la expresan gradualmente de una forma simbólica apropiada para su edad”. (p. 413)

La generalización puede ser expresada de diversas formas. Inicialmente, los niños pueden expresar con palabras las generalizaciones que observan en el mundo y, gradualmente, usar formas más simbólicas. Sin embargo, la idea de trabajar con el desarrollo del pensamiento algebraico desde los primeros años del proceso de escolarización no se centra en el desarrollo de un lenguaje formal con la manipulación de símbolos, sino en las formas de pensamiento en el proceso de producción de significados por el alumno. Cyrino y Oliveira (2011) resaltan que "dependiendo del nivel de experiencia de los alumnos, estas generalizaciones pueden ser expresadas por palabras o por símbolos basados en la observación de patrones o en relaciones funcionales" (p. 102). Fue desde esta perspectiva que se desarrolló la presente investigación.

Carraher et al. (2008), señalan que las “(...) generalizaciones deben surgir en actividades asociadas a situaciones ricas vivenciadas (...) (p. 3, traducción libre ${ }^{1}$ ). Es decir, los alumnos necesitan estar inmersos en contextos problemáticos de modo que utilicen representaciones y el lenguaje natural. En este contexto, el profesor, a partir de su intencionalidad pedagógica, asume un importante papel en la creación de un ambiente de problematización que puede contribuir en los procesos de desarrollo del PA de los alumnos. Tal ambiente es entendido como el movimiento posibilitado por la resolución de problemas y la circulación de significados en el trabajo con los alumnos, lo que implica interacciones entre los alumnos y con la profesora - diálogo, intercambio de ideas, trabajo compartido e intervención de la profesora, de manera que se hace evidente la necesidad de producir cuestionamientos durante las actividades de los alumnos, estimulándolos como investigadores y constructores de su propio conocimiento (Bagne, 2012).

$1[\ldots]$ generalizations need to arise in activities associated with rich experiental situations $[\ldots]$ 
En el ambiente de problematización, la práctica pedagógica del profesor debe tener la intencionalidad de promover el aprendizaje y el desarrollo de los alumnos. El profesor es el responsable de la planeación, de la elección de tareas que prioricen el diálogo y el intercambio de ideas, de la construcción de una cultura de salón de clase enmarcada en la elaboración conceptual y del suministro de herramientas matemáticas que puedan ser usadas como dispositivos de aprendizaje; igualmente, es responsable de garantizar la equidad y la accesibilidad en las clases de matemática, en las cuales, partiendo de aquello que los alumnos ya conocen, las tareas pueden generar avances en el aprendizaje y en el desarrollo (Hiebert et al., 1997).

Nuestro trabajo se apoya en la perspectiva histórico-cultural con los estudios de Lev Vigotski y sus seguidores. Bajo esta perspectiva, el desarrollo humano es un acontecimiento social en el que la apropiación de los bienes culturales proviene de las relaciones personales. Por lo tanto, la educación es entendida como el proceso de humanización de los sujetos, y, esa "humanización" es posibilitada por el proceso de apropiación de los medios culturales que fueron construidos históricamente por la humanidad. La escuela, a su vez, es la institución responsable por el suministro de conocimientos que permiten el desarrollo de las máximas posibilidades; es decir, la escuela tiene como meta la apropiación de los conocimientos científicos por parte de los alumnos - al mismo tiempo que se constituye como la institución responsable de promover la relación entre los conocimientos científicos y los cotidianos/ espontáneos - aquellos que los alumnos traen para el contexto escolar. Los participantes en este proceso [alumnos y profesor] se constituyen por medio de las relaciones sociales y están mediados por los signos e instrumentos. Tales consideraciones traen implicaciones pedagógicas importantes como el reconocimiento de la importancia de la palabra [significado, sentido y significaciones], la intervención del otro y la intencionalidad pedagógica del profesor.

Otro concepto importante de la teoría vigotskiana es la Zona de Desarrollo Proximal o Zona de Desarrollo Próximo - la ZDP. Este concepto es caracterizado por Vigotski (2009) como la distancia entre el nivel de desarrollo real - aquello que el alumno ya logra hacer de modo independiente - y el nivel de desarrollo proximal- aquello que el alumno logra hacer a partir de la intervención del otro. El autor defiende que para una comprensión adecuada del desarrollo hay que considerar, más allá de lo que el niño logra realizar solo - el desarrollo real-, el nivel de su desarrollo potencial/proximal; es decir, aquello que logra realizar con la ayuda del otro. Esta "zona de desarrollo" sería el camino que el sujeto recorre para desarrollar las funciones que están en proceso de consolidación; una vez consolidadas se transforman en el desarrollo real.

De acuerdo con Oliveira (1997) "es en la zona de desarrollo proximal donde la intervención de otros individuos es más transformadora" (p. 61). De este modo, la intervención del profesor y de los niños más avanzados en determinados asuntos puede contribuir en el fomento del desarrollo del otro. Estamos de acuerdo con Freitas (1997) cuando afirma que "la acción compartida, permeando el espacio pedagógico, humaniza el proceso educacional” (p. 308). Sin embargo, hay que tener cuidado con el 
sentido atribuido al término "intervención”, dado que la perspectiva histórico-cultural no sugiere una postura directiva y autoritaria - característica de la enseñanza tradicional. Vigotski (2009) se preocupa por la importancia del medio cultural y las relaciones sociales en el proceso de desarrollo humano.

A partir de estos aportes teóricos, para el trabajo en el salón de clase escogimos tareas que permitan intercambios entre los alumnos: la "ayuda" del otro (profesora o colega) en la realización de las tareas - aquello que logro hacer con la ayuda del otro garantiza la actuación en la ZDP.

A continuación, presentamos la metodología relacionada con la investigación desarrollada. En él, indicamos el contexto de la investigación, el enfoque elegido y la forma en que se produjeron los datos.

\section{Metodología}

La investigación fue realizada a lo largo del año lectivo 2017 en una clase de primer año de Enseñanza Básica - que comprende niños de seis años de edad - compuesta por 26 alumnos de una escuela municipal del interior del estado de São Paulo. Para la documentación de la investigación, las clases de la profesora-investigadora fueron grabadas en video y transcritas, sirviendo de apoyo para la escritura de las narrativas pedagógicas. Partiendo de los presupuestos de la perspectiva histórico-cultural y basándose en los estudios de Góes (2000), en estas narrativas se realiza el análisis microgenético dirigido a los indicios que permiten la interpretación de los episodios observados, permitiendo la aproximación a los procesos referentes al desarrollo del pensamiento algebraico de los alumnos.

Según la definición de Prado et al. (2011), las narrativas pedagógicas son “textos predominantemente narrativos y autobiográficos, escritos para compartir lecciones aprendidas a partir de la experiencia, de la reflexión sobre la experiencia, de la observación de la práctica de los pares, de la discusión colectiva, de la lectura, del estudio y de la investigación” (p. 143). Los autores destacan algunos géneros discursivos que pueden ser considerados como narrativa pedagógica (memoriales de formación, cartas pedagógicas crónicas de lo cotidiano, los testimonios, los diarios, los relatos de experiencia y de investigación, entre otros) que se presentan como dispositivos para que "los educadores documenten lo que hacen, lo que piensan, lo que piensan sobre lo que hacen, así como también sus inquietudes, dificultades, conquistas, y su producción intelectual" (Prado et al., 2011, p. 143). Los autores señalan todavía que todo relato puede ser considerado como narrativa pedagógica desde que posibilite la reflexión. Entonces, la narrativa pedagógica se presenta como un dispositivo para los decires y escritos de los educadores de y en la escuela. No solamente desvelan los dilemas de estos sujetos sino también sus deseos y expectativas, además de revelar posibilidades de reflexión y transformación en/de la práctica pedagógica.

Partiendo de una perspectiva que valoriza las relaciones dialógicas en el salón de clase, los intercambios y las negociaciones de significaciones como proceso de apropiación de conocimientos, hay que pensar 
en las tareas que privilegian tal ambiente de investigación. Entendemos que lo que caracteriza la calidad de la tarea es la coherencia en cuanto al contenido de la misma y las posibilidades de relaciones que pueden ser establecidas a partir de ella -esto último será establecido a partir de los movimientos de intervención en la práctica del profesor y de los alumnos. Por tal razón, adoptamos el término tarea para las situaciones propuestas en el salón de clase, en el sentido de que estas, al entrar en contacto con la cultura social establecida, pueden llegar a ser o no una actividad movilizadora de significados, aunque esto solo puede ser definido en la medida que los alumnos se van sintiendo movilizados, y, efectivamente, se ponen en actividad. El concepto de actividad es concebido por Leontiev (1983) como la acción principal del niño -aquella que promueve el desarrollo y el aprendizaje e involucra los intereses y necesidades de los niños - Tal concepto es ampliado por los estudios de Davidov (1988) que afirma la actividad promotora del desarrollo a partir de una enseñanza "del desarrollo", en la que los conocimientos científicos son tomados como bases del proceso educativo.

A lo largo del año 2017, los alumnos fueron integrados en el desarrollo de una secuencia de tareas enfocada en el pensamiento funcional y en el pensamiento relacional ${ }^{2}$, en la que ambos sucedieron simultáneamente, sin seguir necesariamente un orden o patrón preestablecido. Lo mismo ocurrió con el desarrollo de las tareas, que no fueron propuestas de manera cronológica, dado que no siempre fue posible conciliar la investigación con la docencia porque esta última debe atenerse a las demandas del contenido y de las evaluaciones externas exigidas por la red municipal de educación. El tiempo para cada tarea fue relativo y siempre fue susceptible a lo que los alumnos estuvieran expresando. Había días en los que los diálogos fructificaban, especialmente, los de socialización que siempre tomaron más tiempo; por el contrario, había días en que los alumnos estaban más cansados, lo que hacía que la profesora-investigadora detuviera las discusiones y, de ser necesario, las retomara en otro momento.

Para este estudio, el análisis se centra en la narrativa pedagógica Tarea de las tapas producida por la profesorainvestigadora y que fue desarrollada junto a los alumnos durante la segunda quincena del mes de noviembre de 2017; es decir, al final del año lectivo, cuando los alumnos ya habían vivenciado una serie de tareas que comprendían contextos de secuencias con patrones repetitivos y recursivos. La tarea fue propuesta a los alumnos organizados en parejas o tríos. La selección de los grupos fue realizada por la profesora, que, de manera intencional, buscó posibilitar el intercambio entre las parejas: el alumno que ya tenía el dominio o se aproximaba al código escrito, y que era capaz de hacer la lectura del enunciado con aquel otro que estaba en proceso de apropiación; o el alumno que manifestaba argumentos orales y/o escritos con facilidad con aquel otro que se manifestaba [oralmente] con cierta dificultad. Además, en algunos casos fue necesario considerar aspectos de las relaciones interpersonales entre los niños, dado que algunas parejas, aunque fueran entendidas como una agrupación favorable para intercambios e interacciones, afectivamente no lograban establecer una colaboración y entraban en conflictos que perjudicaban el desarrollo de la tarea.

2 La mayoría de las tareas de esta secuencia hace parte del archivo del "Grupo Colaborativo en Matemática” - Grucomat publicadas en Nacarato y Custódio (2018). 
Para la realización de las tareas y amparada por los estudios de Van de Walle (2009), la profesorainvestigadora siguió el formato de clase en tres fases: antes, durante y después. Cada fase de trabajo tenía una planeación y unos objetivos específicos. La fase antes estaba destinada a verificar si los alumnos entendieron la propuesta de la tarea y la aclaración de las expectativas antes de que los alumnos comenzaran a trabajar - lo que incluye la organización del material y de las parejas/tríos y el levantamiento de los conocimientos espontáneos/cotidianos. De esta manera, después de distribuir la tarea para los niños, la profesora realizaba la lectura del enunciado, ya que la mayoría de los niños aún no estaba alfabetizada. Además, ella buscaba problematizarlos para sondear su interpretación del enunciado propuesto. Durante el transcurso de la secuencia de tareas y a medida que los niños comenzaron a alfabetizarse, la profesora pasó a conceder un tiempo para que intentaran realizar por sí mismos la lectura del enunciado, y después, ella realizaba la lectura junto con ellos, seguida de las problematizaciones.

En la fase durante, los alumnos tenían la oportunidad de resolver los problemas con sus pares; en este momento, la profesora asumía una postura de observación y evaluación; cuando era necesario, formulaba problematizaciones interviniendo en el proceso de los alumnos. Como no alcanzaba a acompañar de cerca a todas las parejas, la profesora fue adecuando las intervenciones, intentando mezclar las problematizaciones en las parejas para cada tarea, destinando algunas para el momento de la socialización.

Finalmente, la tercera fase después, estaba dirigida a la socialización y discusión de las estrategias utilizadas para la resolución de la tarea; esta era una nueva oportunidad para que la profesora pudiera entrar en contacto con las ideas de los alumnos, así como una nueva ocasión para la apropiación de ideas por parte de los alumnos; y era también la fase para la sistematización de los conceptos presentados por la tarea. En esta fase la profesora buscaba socializar las respuestas de los niños y dirigía las problematizaciones hacia las percepciones de regularidad y las generalizaciones de ideas matemáticas; es decir, era el momento de la sistematización de los conceptos matemáticos.

A continuación presentamos la narrativa pedagógica contada en primera persona por la profesorainvestigadora y se presentan los episodios del salón de clase. Tales episodios, que muestran los diálogos de los movimientos en el salón de clase, están organizados por turnos que aparecen con la inicial T seguida de la numeración según la secuencia en la que vayan apareciendo en la narrativa. Para preservar la privacidad de los niños optamos por utilizar nombres ficticios; las intervenciones de la profesora-investigadora, a su vez, aparecen con la inicial $\underline{\mathrm{P}}$ (profesora). La narrativa, por ser un género escrito por la profesora-investigadora, está redactada en la primera persona del singular.

\section{Narrativa pedagógica: Patrones con tapas}

Durante la segunda quincena del mes de noviembre de 2017, los alumnos fueron involucrados en la realización de la tarea Patrones con tapas con el objetivo de que entraran en el movimiento de la 
percepción de regularidad y que desarrollaran estrategias de generalización. La tarea fue realizada durante dos días consecutivos. Primero, fueron suministradas las tapas (reutilizadas de botellas plásticas de gaseosa) a los alumnos para que las parejas pudieran explorarlas libremente -este momento es fundamental para el inicio de una tarea que requiera materiales manipulativos, dado que conocer los objetos, desarrollar curiosidades y experimentar posibilidades hace parte de la planeación y desarrollo de la propuesta. Luego del momento de la exploración, los alumnos recibieron una hoja con el enunciado de la tarea: (Cuadro 1)

\section{Cuadro n. ${ }^{\circ} 1$}

Patrones con tapas

\section{PATRONES CONTAPAS}

\section{TAREA 1}

OBSERVE LA SIGUIENTE SECUENCIA

1. ¿CUÁL SERÍA LA PRÓXIMA FIGURA?

2. ¿CUÁNTAS TAPAS HAY EN CADA FIGURA?

3. COMPLETE LA TABLA DE MODO QUE LOS DATOSii QUEDEN ORGANIZADOS

\begin{tabular}{|c|c|}
\hline NÚMERO DE LA FIGURA & NÚMERO DE TAPAS \\
\hline 1 & \\
\hline 2 & \\
\hline 3 & \\
\hline 4 & \\
\hline$\ldots$ & $\ldots$ \\
\hline
\end{tabular}

\section{4. ¿CUÁNTAS TAPAS HABRÁ EN LA 9 FIGURA?}

5. ¿Y EN LA $20^{\circ}$ FIGURA?

Concedí un tiempo para que los niños pudieran buscar sus estrategias para realizar la lectura del enunciado. Ellos aún estaban en el proceso de apropiación de la lectura y la escritura. En ese momento, la alumna Jade me dijo: "iProfesora, pero, nosotros no tenemos dados!" - haciendo referencia al término “datos”ii en el tercer ítem del enunciado, relacionando el término al objeto utilizado para contar 
en juegos y pasatiempos: el hexaedro con las caras numeradas de 1 a 6. Esta intervención me hizo reflexionar acerca de la importancia de ese primer momento de análisis realizado por los niños, así como también acerca de lo importante de estar atenta a las significaciones de los alumnos frente a los enunciados, ya que los alumnos pueden atribuir diferentes sentidos al texto dando cabida a la negociación de significaciones para el desarrollo de la propuesta. Es importante que los niños tengan tiempo suficiente para pensar, elaborar sus hipótesis y hablar sobre ellas, ya que no siempre atribuyen el mismo significado que la profesora asigna al enunciado de la tarea.

Bakhtin (1988) explica que los sentidos de una palabra no existen en sí mismos como algo dado. Los sentidos son elaborados en las enunciaciones y estas, a su vez, hacen parte de un diálogo social. El enunciado "es el territorio común entre el locutor y el interlocutor" (Bakhtin, 1988, p. 113) - y todo enunciado se refiere a por lo menos dos sujetos: procede de alguien y se dirige a otro. Entonces, los sentidos llevan marcas de las condiciones sociales en las cuales son producidos, y por eso, ellos son parcialmente "nuestros" y parcialmente del "otro" en un proceso de interacción entre los locutores. El sentido es producido y "significado" siempre en la interacción de las voces! Para formular el sentido expresado por "datos" en el enunciado utilicé el contexto de una tarea realizada anteriormente por los alumnos, en la que también fueron involucrados en la construcción de una tabla con los "datos" de la secuencia; buscando lo que Radford (2008) llama "movimiento de iconicidad" caracterizada por la disposición de un repertorio de experiencias importantes que permiten retomas y (re)significaciones por parte de los alumnos.

Después del momento inicial hago la lectura colectiva con los alumnos, nuevamente negociando las significaciones de los niños y hasta discutiendo algunas preguntas formuladas por ellos. A continuación, Maíra me llama y afirma:

Episodio 1 - Descubrimiento de Maíra

T01 Maíra: Profesora, yo encontré la del 9. 9+9 da 18!

T02 P: ¿¿ en qué pensaste para descubrirlo?

T03 Maíra: Pensé en la del 5

T04 P: ¿Y qué hicimos en la del 5 ?

T05 Maíra: Pensamos en cuánto da $5+5$.

T06 P: ¿्Y si pienso en la 20?

T07 Maíra: 40!

T08 P: ¿् Y si pienso en la 21?

T09 Maíra: [pensativa] voy a pensarlo...

T10 P: Mira, la 20 da 40! ¿̇ la 21?

T11 Maíra: ;41!

El episodio muestra indicios de la síntesis del análisis realizado por Maíra frente a la regularidad de la secuencia: "Profe, encontré la del 9. 9 + 9 da 18" (T01) - la alumna utiliza la adición del número 
del término $(n+n)$, para determinar la cantidad de tapas en la figura 9. Pero, ¿cómo llegó ella a esa percepción? "Mire, profesora, a partir de lo que bicimos colectivamente en el término 5 ahora yo logro realizar, sola, el término 10. "Es decir, lo que antes era realizado con la ayuda del otro, pasa a ser realizado de manera autónoma. En este sentido, pienso que las acciones de la tarea están, de hecho, actuando en la ZDP de los niños (Vigotski, 2009).

En la secuencia, reto a la alumna a pensar sobre otros términos (T06; T08). ¿Será que su "regla” era válida para todos los términos de la secuencia? La alumna no mostró dificultades para determinar la cantidad de tapas de la figura 20 (T07) evidenciando su apropiación de la estrategia de generalización $(n+n)$. Sin embargo, cuando le pregunto sobre el término 21, nos deparamos con un nuevo desafío: cómo realizar la adición $21+21$. Le sugiero ir un paso atrás, cuando pensábamos en la adición 20 + 20 (T10). Entonces, Maíra afirma que el resultado de esa adición sería 41 (T11). A pesar de la incoherencia, pienso que su discurso tiene una lógica válida porque al final, ¿̇no había yo aumentado una unidad al número de la suma anterior? Sin embargo, era necesario considerar que se trataba de una unidad para cada número de la adición, lo que resultaría en un total de 42 tapas. Cabe destacar que la suma con números de mayor magnitud aún estaba en proceso de construcción con los niños, ya que no forma parte del currículo adoptado en el sistema escolar. Los estudiantes estaban construyendo estrategias de cálculo mental y Maíra estaba tratando de encontrar la suma de esta adición; sin embargo, ella en ese momento no pudo resolver la suma, pero llegó a una ley de formación a partir de un discurso de argumentación (Blanton y Kaput, 2005).

Para dar por terminada la tarea, propongo la socialización de las estrategias utilizadas por los niños. Para algunos - que estaban comprometidos con el análisis y búsqueda de una generalización - este era un momento de síntesis, pero, para aquellos niños que aún estaban en proceso de apropiación de la tarea, o incluso, en el proceso de apropiación de la regularidad de la secuencia, era una nueva oportunidad de (re)significación, y, en consecuencia, de aproximación a la propuesta de la tarea. Entonces, los niños que aun no estaban movilizados por la búsqueda de regularidades, podrían hacerlo con mi ayuda y la de los compañeros con más experiencia.

Episodio 2 - Aumentado de 2 en 2

T01 P: ¿Qué tipo de secuencia es esta?

T02 Jade: Va saltando de 2 en 2.

T03 Pedro: Aumenta de 2 en 2.

T04 P: ¿Va saltando o va aumentando de 2 en 2?

T05 Alunos: Aumentando

T06 Antônio: de 3 en 3...

T07 P: ¿Cómo así, Antonio?

T08 Antonio: Alli va aumentando de 3 en 3.

T09 P: ¡Ven y explicame! 
T10 Antonio: [va hasta la pizarra y señala las dos columnas de la figura 3]

T11 P: Ah, entiendo... ¿̇En qué pensó Antonio, niños?

T12 Gabriela: En el número de la figura...

T13 Pedro: También está saltando de 2 en 2 porque aquí [va hasta la pizarra y señala los espacios entre las figuras] se saltó el 3 , aquí se saltó el 5 , aquí el $7 \ldots$

T14 P: Maira observó algo... jhabla Maira!

T15 Maíra: Es una secuencia de números pares...

T16 Antonio: El 2 es par!

T17 P: ¿Será que Maíra tiene razón?

T18 Antonio: Si, porque el 2 es par, el 4 ...

T19 Jade: El 6 es par, el 8 es par, el 10 es par...

T20 P: Ahora quiero entender cómo hago para saber cuantas tapas hay en la figura 9.

T21 Lia: $; 9$ !

T22 P: $\dot{6} 9$ ?

T23 Lia: No... ;8!

T24 P: ¿̇ú piensas que hay una menos, como en aquella tarea $?^{33}$ [señala la cartelera en la pared ${ }^{4}$ ]

T25 Lia: ;11!

T26 Jonathas: $; 20$ !

T27 Alumno: ;18!

$\mathrm{Al}$ inicio del episodio, me doy cuenta de que los niños van negociando significados para lo que sucede en la secuencia: "¿Será que ella está aumentando o saltando de 2 en 2 ? ¿ंHabría alguna diferencia entre esos términos?” (T02, T03, T05) - iperdíla oportunidad de preguntarles!. Sin embargo, al reflexionar acerca de esta pregunta, me acuerdo de las tareas en las que utilizábamos la recta numérica - el análisis de los episodios también me estaba posibilitando el movimiento de iconicidad (Radford, 2008) - En aquellas tareas dábamos "saltos" entre los números, sin embargo, la dirección del "salto" (derecha o izquierda) determinaba si "aumentaba" o "disminuía”; así que, considerando este contexto, sí habría diferencia en el uso de los términos!

¿La regularidad de la secuencia puede ser establecida, en un abordaje aritmético, de "2 en 2" o de "3 en 3"? Ese era el levantamiento de los niños y yo estaba movilizada para descubrir significaciones. Ante la afirmación de Antonio (T06), le pido una explicación para su hipótesis (“3 en 3”). A partir de su explicación, en la que señala a las "partes" de la figura, entendí que centrando su análisis en la figura 3, su intervención hacía referencia a que el número de la figura se repetía en la cantidad de tapas dispuestas en cada columna. Sería imposible mi aproximación a la significación atribuida

3 Una tarea realizada anteriormente por el grupo que comprendía una secuencia recursiva usando palillos

4 Al final de cada tarea de patrones recursivos hicimos un registro de los resultados [tabla, estrategias de generalizaciones y ley de formación] en hojas de papel marrón y las fijamos en la pared para futuras consultas. 
por el alumno si yo estuviera partiendo de una interpretación totalmente subjetiva sin considerar las significaciones elaboradas por ellos. Los alumnos tienen mucho "qué decir" sobre "lo que dicen"; y, por otra parte, la profesora tiene mucho "que entender" sobre "lo que entiende".

$¿ Y$ no existen muchas maneras de observar la regularidad en la secuencia? Observemos el movimiento de Pedro (T13): él pasa a considerar los "números impares que la secuencia se saltó"; los que estarían en los espacios en blanco entre una figura y otra: 3, 5 y 7 . Tuve que retomar la secuencia para entender su raciocinio y considerar la regularidad señalada por el alumno. Los niños me ayudan a observar cosas que no podría observar yo sola. ¡¿ ¿ cuántas cosas más pasarán desapercibidas por no solicitarles a mis pequeños que expliquen lo que están pensando?! Me doy cuenta de que mi intervención busca involucrar a toda la clase, o a un mayor número de niños, en el movimiento de pensar junto a aquellos que estaban intentando encontrar una regularidad para la secuencia. En el turno 14, llamo a Maíra para que comparta su idea con los colegas. En un principio, yo seguía pensando acerca del "descubrimiento" que había compartido conmigo anteriormente, y, por tanto, me imaginé que ella iba a explicar cómo sumar los números para la obtención de la cantidad de tapas. Sin embargo, me sorprendo con una nueva forma de observar la regularidad: "Es una secuencia de números pares..." Me doy cuenta de que la alumna rompe con el abordaje aritmético inicial y parte para otro tipo de abordaje, formulando una nueva hipótesis. Pero, ¿qué estaba considerando ella? ¿La suma de las tapas de cada figura?¡¡ Si así fuera, su respuesta estaría correcta! Sin embargo, y, ¿`i ella estuviera considerando otro aspecto en su análisis? ¡Perdí la oportunidad de preguntarle!.

¿Y cómo reaccionan sus colegas ante su afirmación? Van a buscar indicios para validar su hipótesis: “El 2 es par!", “... el 4"; “El 6 es par, el 8 es par: el 10 es par...”.Entonces, el grupo validó la hipótesis de su colega. A partir de la discusión, busco (re)orientar el análisis del grupo en busca de la cantidad de tapas en la figura (T20). Noto algunas iniciativas basadas en "intentos y errores" (T21, T23, T25, T26). Me llama la atención que, al comienzo, la alumna Lia expresa la cantidad de 9 tapas (T21) y, después de ser cuestionada, señala la cantidad de 8 tapas (T23) - aquí, yo entendí que ella hacía referencia a una tarea anterior en la cual realizábamos la substracción (-1) en cada término. Sin embargo, terminé por equivocarme al "inducir" el hecho a la alumna, afirmándola sobre mi percepción. Lo correcto habría sido dejar que la alumna, por sí misma, evidenciara su significación con la secuencia, y, en lugar de cuestionarla con “¿Tú piensas que hay una menos, como en aquella tarea?”; debería haber utilizado preguntas que fueran más abiertas: “¿Cómo asi? ¿̇Por qué dijiste 8?".

Buscando (re)organizar el análisis de los niños para que consideraran el número de la figura en busca de una estrategia de generalización, modifico mi pregunta:

Episodio 3 - Rescatando ideas importantes

T01 P: ¿¿Será que si pienso en el número de la figura, eso me ayuda a descubrir la cantidad de tapas?

T02 Alumnos: ¡No!

T03 P: Miren... [señala la figura 2] ¿̨el número 2, tiene alguna relación con la figura? 
T04 Alumnos: ;No!

T05 P: ¿̨Será? Antonio habia dicho que si habia relación.... ¿̇Qué dijiste en ese momento?

T06 Antonio: iiSi hay!!

T07 $\mathrm{P}_{\grave{c}}$ Cuál es la relación?

T08 Antonio: La relación es que se está repitiendo...

T09 P: ¿Qué se está repitiendo? Miren, Jonatas también lo identificó. ¿̇Te acuerdas de cuando pregunté sobre el 5 ? Dijiste que era solo tomar el $5+$ esto [gesticula]

T10 Jonatas: [va hasta la pizarra y señala el número de la figura y lo asocia a la cantidad]. Ese dos está aquí, miren...

T11 P: ;Miren lo que Jonatas descubrió! Que este 2 [número de la figura] se repite aquí [número de tapas]. ;Figura 2, 2! [señala la cantidad]; ;Figura 1,1! [señala la cantidad de tapas]; įंFigura 3?!

T12 Alumnos: ; 3 !

T13 P: $:$ Y en la figura 9?

T14 Alumnos: 9....j!

T15 P: Entonces, para que descubramos cuántas tapas hay en una figura...

T16 Jade: Vamos a necesitar el número que hay... [señala la secuencia de la pizarra]

Voy llamando a los alumnos que estaban intentando encontrar una regularidad en la secuencia. (T05, T09). Sin embargo, únicamente logro involucrar a los alumnos en esta discusión porque ya conocía sus producciones. El "caminar" por la salar mientras los alumnos realizan las tareas es fundamental para entrar en contacto con lo que ellos están produciendo, para realizar problematizaciones que puedan auxiliar en las diferencias cualitativas de sus significaciones, para actuar en la ZDP (Vigotski, 2009) y para seleccionar sus producciones para el momento de la socialización

Percibo mi movimiento de intervención en busca del rescate de los señalamientos realizados por los alumnos [durante el trabajo en pareja] que yo consideraba que podían contribuir en las discusiones y negociaciones colectivas dirigidas a la generalización de la secuencia. Un ejemplo es el turno 5, en el que llamo a Antonio para compartir nuevamente su percepción con los compañeros - destacada en el episodio anterior [“de 3 en 3"] -. En este sentido, los alumnos actúan en la intervención del proceso de aprendizaje de sus pares, aun cuando la intervención, en momentos como estos, necesite de mi mirada y destaque en el sentido de retomar las intervenciones de los alumnos y de animarlos a hablar sobre sus percepciones, hipótesis y estrategias.

Todavía sobre este momento del episodio, noto cuánto se ha apropiado Antonio de la circulación de ideas en el salón de clase y cómo (re)organiza su comunicación: "La relación es que se está repitiendo..." - dado que, anteriormente, él dijo “...aumentando de 3 en 3" apoyándose en la situación real de la secuencia. No hay cómo determinar si el alumno utiliza esa expresión asignándole el mismo significado que yo le atribuyo a los conceptos involucrados, dado que él podría estar apenas reproduciendo lo que yo dije. Sin embargo, "primero, el niño es guiado por la palabra del otro y, después, él mismo utiliza las 
palabras para orientar su pensamiento" (Góes y Cruz, 2006, p. 33). Además, Vigotski (2009) afirma que los signos ejercen el papel de mediadores en el proceso de elaboración conceptual ${ }^{5}$, y entre los muchos signos que pueden desempeñar este papel, la palabra ocupa un lugar central, dado que ella está entendida por el autor como medio principal de conceptualización.

Entonces, aun si Antonio estuviera "reproduciendo" lo que yo dije para exponer sus ideas, podría considerar que él estaba mostrando indicios de su proceso de apropiación, y, en consecuencia, de elaboración conceptual; tal apropiación, en el transcurso de su desarrollo será utilizada para orientar su pensamiento, transformando y reelaborando elementos de un contexto "interpersonal" en el contexto "intrapersonal". Por tal razón, "todas las funciones en el desarrollo del niño aparecen dos veces: primero a nivel social, y, después, a nivel individual; primero, entre las personas (intersicológico), y, después, en el interior del niño (intrasicológico)"(Vigotski, 2007, p. 57).

Además, el gesto como una de las múltiples expresiones del lenguaje desempeña un papel significativo en la comunicación de las ideas, hipótesis y análisis de las secuencias. Este lenguaje gestual es revelado tanto por mí - para ejemplificar el duplo del número para los niños (T11) - como por las manifestaciones de los alumnos, como en el caso de Jonatas, quien se desplaza hasta la pizarra para señalar los términos de la secuencia y así explicar su idea a sus compañeros (T10). Vigotski (2007) apunta al gesto como la primera forma de representación del significado. Si, por un lado, el lenguaje oral posibilita la explicación por medio de palabras, el lenguaje gestual, que a veces lo complementa o incluso lo sustituye, busca ejemplificar estas palabras.

Igualmente, Radford (2012) afirma que el uso del ritmo y los gestos del profesor -también apropiados por los niños- abre posibilidades para que los niños echen mano de diferentes recursos en busca de la generalización, que pueden ser apropiados y utilizados no solo en la resolución de retos actuales, sino que también actúan como experiencias que pueden ser tomadas como referencias importantes (movimiento de iconicidad) en otras secuencias. Por medio de diferentes instrumentos semióticos (habla, gesto, sonoridad), los niños crean sus significaciones. Entonces, tales instrumentos aproximan a los niños al proceso de elaboración conceptual. Es importante observar que el discurso de Antonio da una nueva dirección al análisis de la secuencia, dado que a partir de su intervención "3 en 3", que analizamos y posteriormente vamos a usar en la generalización, dejamos de introducirnos en un abordaje de pensamiento recursivo - propuesto por la tarea - y pasamos a analizar la dependencia entre el número de la figura y el total de tapas de cada término. Hay aquí una (re)orientación de nuestro análisis. Me incluyo en este proceso, pero solo tomo conciencia de este movimiento al reflexionar sobre el episodio.

\footnotetext{
5 "El concepto es imposible sin palabras, el pensamiento en conceptos es imposible fuera del pensamiento verbal; en todo este proceso, el momento central, que tiene los fundamentos para ser considerado causa corriente de la maduración de conceptos, es el empleo específico de la palabra, el empleo funcional del signo como medio de formación de conceptos". (Vigotski, 2009, p. 170)
} 
Apostando por la discusión colectiva como un importante dispositivo de aprendizaje, cuestiono nuevamente a la clase sobre la estrategia de generalización de la secuencia. Algunos alumnos no se manifiestan oralmente tanto como los otros, y por esto, siempre busco "una nueva oportunidad de incorporar a toda la clase" en el movimiento.

Episodio 4 - Sistematizando la discusión

T01 P: Entonces yo tengo que hacer el número [mientras habla, registra en la pizarra la palabra número]. ¿El número de qué?

T02 Alumno: $; 10 !$

T03 P: ¿El 10 es el número de qué?

T04 Jade: ;No, profe! ;Tienen que ser los números!

T05 P: Aqui, ¿¿qué pensamos? [señala la figura 2]. ¡En el número de la figura! Entonces, tengo que poner el número de la figura más ...?

T06 Jade: ;Más el número!

T07 P: ¿¿ué número?

T08 Jade: Como el 33... $33+33$ [gesticulando con las manos una columna de 33 y otra de 33]

T09 P: ¿̇Entonces es el número de la figura más el número de la figura?

T10 Jade: Es, lo que Antonio nos enseñó...

T11 P: ¡Entonces, vamos a crear una regla para esa secuencia! Cuál es la regla?

T12 Jade: Se va repitiendo. $1+1 ; 2+2 ; 3+3 \ldots$ [mientras habla gesticula la organización de las figuras]

Como observamos, terminamos la tarea con una ley de formación de la secuencia elaborada por medio de la intervención y de la discusión colectiva. En este punto, destaco la importancia de la socialización de las ideas de los alumnos, particularmente en el trabajo con niños al inicio de la escolarización, como es el caso de mi clase. Ellos se manifiestan con más facilidad por medio de la oralidad, dado que la mayoría está recién alfabetizada y domina con más facilidad el lenguaje oral. Además, la socialización permite al profesor la toma de decisiones frente a las significaciones que los alumnos expresan y posibilita la sistematización de los conceptos obtenidos de la tarea.

Llegamos a la generalización por medio de la adición de los números de la figura " $n+n$ " — si los niños ya se hubieran apropiado del concepto de multiplicación, podríamos pensar en "2n"-. Noto que los niños se valen del uso de ejemplos para comunicar la estrategia de generalización: "Por ejemplo el 33... $33+33$ ” (T08) y el gesto indica la repetición del número en la figura. Así, entiendo que, si por un lado el lenguaje oral asume la función de designar, nombrar y explicar, por otro lado, el lenguaje gestual ejemplifica!, en un movimiento en el que el lenguaje oral y gestual se complementan (Pinto, 2010).

$\mathrm{Al}$ retomar el diálogo en los turnos 09 y 10, "Entonces es el número de la figura más el número de la figura?; Es lo que Antonio nos enseñó..." y reflexionar sobre ello, noto que, durante la clase, soy una figura importante en la intervención de los procesos de aprendizaje de los alumnos, pero, no solamente eso... 
mi mirada atenta (y, a veces, desatenta) busca posibilidades de intervenir en el proceso de elaboración conceptual de los niños. Traigo tareas, creo situaciones, incentivo participaciones, provoco, llamo la atención... Todo esto para poner en evidencia a los verdaderos protagonistas de la clase: ¡los niños! Protagonistas de la clase; protagonistas del proceso de elaboración conceptual de sí mismos y del colectivo del salón de clase. El lugar del protagonismo, de la construcción, del intercambio, de la participación; el lugar significativo, el lugar de la pertenencia (Ricouer, 1989). Es verdad, Antonio enseñó y nos enseñó a todos, pero, no podemos olvidar que Antonio, al enseñar, también aprende con nosotros. Nuestras voces se mezclan, se (trans)forman al encontrarse; nos retornan [aún siendo nuestras voces] (re)significadas. El desarrollo del PA se da en el intercalado de las mezclas de voces.

Para finalizar, integré a los alumnos en la construcción de la tabla donde organizamos los datos de la secuencia.

Tabla n.o 1

Secuencia de las Tapas I

\begin{tabular}{c|c|c}
\hline NÚMERO DE LA FIGURA & $\begin{array}{c}\text { LEY DEL 1ER ANO } \\
\text { NÚMERO DE LA FIGURA + } \\
\text { NÚMERO DE LA FIGURA }\end{array}$ & NÚMERO DE TAPAS \\
\hline 1 & $1+1$ & 2 \\
\hline 2 & $2+2$ & 4 \\
\hline 3 & $3+3$ & 6 \\
\hline 4 & $4+4$ & 10 \\
\hline 5 & $5+5$ & 12 \\
\hline 6 & $6+6$ & $\ldots$ \\
\hline$\ldots$ & $\ldots$ & 24 \\
\hline 12 & $12+12$ & $\ldots$ \\
\hline$\ldots$ & $\ldots$ & $\ldots 0$ \\
\hline 40 & $40+40$ & 100 \\
\hline
\end{tabular}

La ley de formación "número de la figura + número de la figura" $(n+n)$, a pesar de que surge en las discusiones de la socialización, fue sistematizada por medio de la construcción de la tabla. Aquí, tuvimos la oportunidad de una nueva visualización de la secuencia. Salimos del contexto figurativo para el contexto de la tabla, en el cual existe la posibilidad de una [nueva] lectura y visualización de la secuencia. Pienso que este proceso de salir de lo figurativo a lo numérico, y lo contrario también [salir de lo numérico a lo figurativo] posibilita la (re)significación para los niños, ampliando el entendimiento y reconocimiento de las regularidades y favoreciendo el proceso de generalización. 
Esta fue una generalización construida por los alumnos. Otra posibilidad que se me ocurrió al mirar hacia atrás en esta secuencia sería la recursividad de sumar 2 al resultado anterior, lo que generaría otra ley de formación: $2+2$. (n - 1). Así, la secuencia propuesta era recursiva, pero la generalización producida por los alumnos no.

Reconozco la importancia de utilizar la tabla para el proceso de generalización. Hiebert et al. (1997), señalan que:

la actividad matemática requiere del uso de herramientas, y las herramientas que usamos influyen en la manera como pensamos sobre la actividad. Otra manera de decirlo es que las herramientas son un recurso y soporte esencial para la construcción de la comprensión matemática, y las herramientas que los alumnos usan influyen sobre los tipos de entendimientos que desarrollan. (p. 10, traducción libre ${ }^{6}$ )

Las autoras argumentan que las herramientas matemáticas pueden ayudar a los niños a hacer cosas que no podrían hacer solos; es decir, actúan como mediadoras en el aprendizaje de los niños. Entiendo la tabla como una herramienta de mediación en el proceso de análisis y generalización de las secuencias. Sin embargo, el uso (significativo) de la tabla se da en un proceso de negociación de significaciones junto a los niños. ¡Cuando decidí incluir una tabla en el enunciado no pretendía que ese uso "significativo" sucediera a partir de esa tarea! Entiendo ese "uso" como algo a ser conquistado a partir de un proceso: en el transcurso de las negociaciones de las significaciones, en el transcurso de las secuencias de las tareas. En el contexto de mi salón de clase aún es una actividad que necesita ser actuada en la ZDP (Vigotski, 2009). Al analizar los registros de los niños percibo que atribuyeron diferentes sentidos a la tabla, pero aún no se habían fijado en el registro solicitado por el enunciado e incluyo aquí a los niños que optaron por no realizar ningún registro. Como ejemplo de este proceso tomo el registro de Pedro:

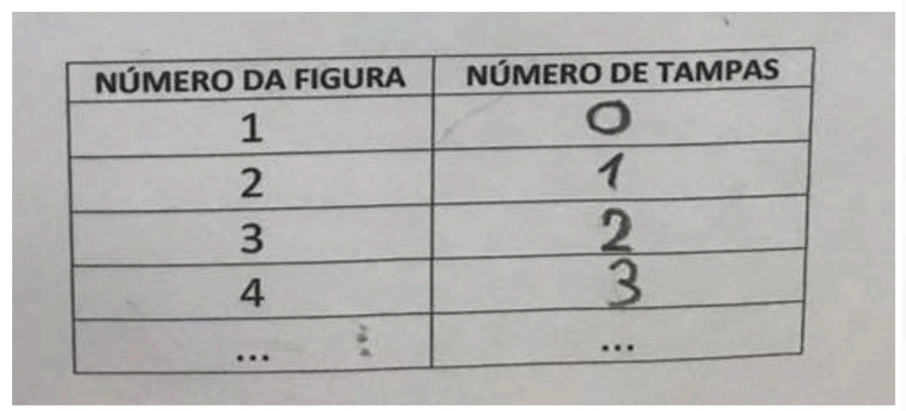

Figura n. ${ }^{\circ}$ 1. Registro en la tabla del alumno Pedro. Fuente: Archivo de la investigadora

6 "Mathematical activity requires the use of tools, and the tools we use influence the way we think about activity. Another way to say this is that tools are an essential resource and support for building mathematical understanding, and the tools students use influence the kinds on understandings they develop" 
"Profesora, ¿qué hago con ese espacio en blanco de aquí? ¿Será que tengo que escribir los números en orden? ¿Será que tengo que copiar el "modelo"? - El alumno echa mano del registro de la secuencia numérica, sin fijarse en lo que la columna indicaba. Otros repitieron el número que aparecía en la primera columna. Sin embargo, en el momento de la socialización logro retomar las cuestiones de significación importantes para la tabla, cosa que ellos aún no podían realizar solos, pero que podían hacer con mi ayuda. “¡Y no hay problema!” - entiendo esa aceptación mediante mi proceso de autoformación, dado que en un pasado no muy distante, estaría angustiada con "lo que no funcionó” en el desarrollo de la propuesta. Pues, estaba amparada por mi planeación, por mi intencionalidad pedagógica y (más aún) por mis reflexiones sobre la teoría [aliada a la práctica] que me permiten la comprensión y aceptación del proceso (marcado por altas y bajas). Aquí, entiendo que esa comprensión es importante pero no suficiente... Es necesario un movimiento "más allá"; ¡es necesaria la aceptación! Comprender los procesos de enseñanza y aprendizaje (en mi caso, a la luz de los presupuestos de la perspectiva histórico-cultural) y la aceptación (de la práctica) en que se involucran movimientos, tiempos y "resultados" que rompen con los modelos tradicionales de enseñanza... y no hay problema si los niños todavía no le atribuyen sentido al uso de la tabla como un dispositivo importante para la organización y lectura de los datos; por medio de mi trabajo intencional ellos tendrán nuevas oportunidades de (re)significación — que no dependen del azar, sino de una planeación intencional.

\section{Análisis de la narrativa de la profesora-investigadora}

Los episodios analizados en la narrativa de la profesora-investigadora indican cómo los alumnos, desde el inicio de la escolarización, son capaces de hacer generalizaciones por medio del lenguaje materno. Esto corrobora las investigaciones tomadas como referencia para este trabajo (Mason, 2007; Cyrino y Oliveira, 2011; Radford,2012, 2013,2014). Sin embargo, hay que considerar algunas características de la clase de la profesora- investigadora: hay una intencionalidad pedagógica, con objetivos explícitos para la tarea propuesta, la tarea está cuidadosamente seleccionada e incluida en una secuencia más amplia que permitió un movimiento de iconicidad (Radford, 2008), ya que los alumnos lograron traer a la discusión elementos conceptuales ya apropiados en tareas anteriores. Las tareas son problematizadoras (Hiebert et al., 1997) posibilitando que los discursos matemáticos sean producidos, las interacciones entre los alumnos y la intervención de la profesora posibilitaron la circulación de ideas matemáticas y la generalización de la secuencia; además, fueron utilizados múltiples lenguajes (oral, escrito, gestual) y la profesora echó mano de una importante herramienta para la representación: la tabla, que favorece la percepción de regularidades en la secuencia y su ley de formación. Finalmente, la profesora creó una cultura social en el salón de clase (Hiebert et al., 1997) enmarcada en la problematización y en la cual, los alumnos fueron protagonistas de su propio proceso de aprendizaje. En esta cultura, su clase se basa en las fases de la clase (Van de Walle, 2009), la fase antes que implica la planificación y el inicio de la tarea, la fase durante la realización de la tarea en la que la profesora-investigadora sigue las discusiones en grupo y selecciona los argumentos de 
los estudiantes y, finalmente, los lleva a la fase después en la que tiene lugar la discusión colectiva, estos argumentos se validan o no y permiten la elaboración conceptual. Se puede decir que en estos episodios hay indicios de un PA en desarrollo.

Mirando en retrospectiva el proceso de generalización de los estudiantes, consideramos que la profesora-investigadora, al ofrecer las tapas para la construcción de la secuencia, pudo haber realizado intervenciones que nos ayudaran a generalizarla de forma recursiva a través de la adición de 2 unidades al término anterior. Los alumnos argumentaron usando razonamiento aditivo, ya que la multiplicación aún no se había introducido en el aula. Este habría sido un momento oportuno para tal introducción. Esto refuerza la importancia de la investigación sobre la propia práctica de enseñanza cuando el investigador registra los datos para su posterior análisis. La comprensión a posteriori de esta posibilidad le trajo nuevos conocimientos pedagógicos a la profesora-investigadora.

Igualmente importante fue la apropiación por la profesora-investigadora de los conceptos clave de la perspectiva histórico-cultural, tanto para la organización de esta cultura en el salón de clase como para el propio proceso de análisis de los episodios en la narrativa producida. Consciente de conceptos como desarrollo, aprendizaje, mediación, interacción, el papel del otro y de la palabra, la elaboración conceptual y el lenguaje, ella va orquestando el salón de clase, permitiendo que los alumnos expresen sus puntos de vista, argumenten y vayan hasta la pizarra para explicar su raciocinio. Al final, son alumnos incluidos en una auténtica actividad matemática.

La narrativa de la profesora-investigadora también muestra indicios de sus aprendizajes; al colocarse a la escucha, ella se sorprendió en algunos momentos con las observaciones elaboradas por los alumnos, las cuales la llevaban a raciocinar y validar, evidenciado la imprevisibilidad en un salón de clase cuando los alumnos pueden expresar sus ideas.

Esta es una investigación de la propia práctica de la profesora-investigadora y en su narrativa va revelando su asumir de la investigación como postura. Este concepto fue desarrollado por CochranSmith y Lytle (2009). Según las autoras:

La investigación como postura está fundamentada en los problemas y contextos de la práctica, en primer lugar, y en las formas con las que los profesores en ejercicio teorizan colaborativamente, estudian y actúan sobre esos problemas para cumplir con los intereses del aprendizaje y para ofrecer mejores oportunidades de vida a los estudiantes y a sus comunidades (Cochran-Smith y Lytle, 2009, p. 123, traducción libre ${ }^{7}$ ).

7 Inquiry as stance is grounded in the problems and contexts of practice in the first plane and in the ways practitioners collaboratively theorize, study, and act on those problems in the best interests of the learning and life chances of students and their communities. 
Las autoras argumentan que los profesores:

Están profundamente comprometidos con el trabajo de enseñanza y aprendizaje y saben algo sobre ese trabajo (...) son capaces de generar y criticar el conocimiento, descubrir cómo usar (o no) el conocimiento generado por otros, mejorar la práctica e incrementar las oportunidades en la vida de los estudiantes. (Cochran-Smith y Lytle, 2009, p. 125, traducción libre).

Esta es una característica de la profesora-investigadora que, desde su graduación viene participando activamente en grupos de estudio e investigación y está comprometida con la investigación de su propia práctica.

De esta forma, identificamos su preocupación por la organización del salón, la utilización de formas de registro (la grabación seguida de narrativa) y por cómo entrecruza los sucesos en el salón de clase con el movimiento de pensamiento de los alumnos y las voces de los autores tomados como referencia. Ella se revela en proceso de formación considerando la incompletitudiii del ser humano y, así, analiza con coherencia los momentos en los que podría haber sido más inquiridora con los alumnos; ella tuvo la osadía y logró analizar, lo que podría haber ocurrido de otra manera si ella no hubiera tenido sus clases grabadas en video, ya que esta es una excelente herramienta para investigaciones en el salón de clase. Incluso con algunos límites, principalmente porque la mirada es selectiva y la profesora-investigadora no contaba con un auxiliar para ayudarla en la grabación, la videograbación permite que el investigador recurra a este registro en varios momentos y pueda captar las imágenes y gestos de los interlocutores. Por medio del análisis de las grabaciones en video es posible desvelar el escenario que se estableció en el salón de clase, ya que es posible ir adelante y atrás en el registro para analizar el movimiento de producción y (re)significación de los alumnos, y, al mismo tiempo, analizar las intervenciones que fueron realizadas durante este proceso.

$\mathrm{Al}$ asumirse como profesora-investigadora de su propia práctica, ella posibilita el desarrollo de sus alumnos que, con apenas seis años e iniciando el proceso de alfabetización, logran presentar argumentos matemáticos y muestran indicios de generalizaciones algebraicas.

\section{Referencias}

Bagne, J. (2010). A elaboração conceitual em matemática por alunos do $2^{\circ}$ ano do ensino fundamental: Movimento possibilitado por práticas interativas em sala de aula. (Dissertação de Mestrado). Universidade São Francisco, Itatiba.

Bakhtin, m. (1988). marxismo e filosofia da linguagem. problemas fundamentais do método sociológico na ciência da linguagem. são paulo: hucitec.

Blanton, M. L., \& Kaput, J. J. (2005) Characterizing a classroom practice that promotes algebraic reasoning. Journal for Research in Mathematics Education, 36(5), 412-443. 
Carraher, D. W. et al. (2008). Early algebra and a mathematical generalization. ZDM Mathematics Education, 40, 3-22.

Cochran-Smith, M., \& Lytle, S. L. (2009). Inquiry as stance: Practitioner research for the next generation. New York: Teachers College Press.

Cyrino, M, C. C. T., \& Oliveira, H.M. (2011). Pensamento algébrico ao longo do Ensino Básico em Portugal. Bolema. Rio Claro (SP),24(38), 97-126.

Davidov, V. V. (1988). La enseñanza escolar y su desarrollo psicológico. Moscu: Editorial Progresso.

Freitas, M. T. A. (1997). Nos textos de Bakhtin e Vygotsky: um encontro possível. In: Brait B, organizador. Bakhtin, dialogismo e construção do sentido. Campinas (SP): Editora da Unicamp.

Góes,M.C.R.(2000,Abril). A abordagem microgenética na matriz histórico-cultural: uma perspectiva para o estudo da constituição da subjetividade. Cadernos Cedes, XX(50).

Góes, M. C.R., \& Cruz, M. N. (2006). Sentido, significado e conceito: notas sobre as contribuições de Lev Vigotski. Pro-Posições, 17(2), 31-45.

Hiebert, J. et al. (1997). Making sense: Teaching and learning mathematics with understanding. Portsmouth: Heinemann.

Leontiev, A. N. (1983). Actividad, conciencia y personalidad. Havana: Editorial Pueblo y Educación.

Mason,J. (2007). Making use of children powers to produce algebraic thinking. In J.J. Kaput, D. W. Carraher \& M. L. Blanton (Eds.). Algebra in the early grades (pp. 57-94). New York: Lawrence Erlbaum Associates.

Mestre,C.M.M.V.(2014). O desenvolvimento do pensamento algébrico de alunos do $4 .^{\circ}$ ano de escolaridade: uma experiência de ensino. (Tese de Doutorado). Universidade de Lisboa, Lisboa.

Ministério da Educação. (2012). Elementos conceituais e metodológicos para definição dos direitos de aprendizagem e desenvolvimento do ciclo de alfabetização $\left(1^{\circ}, 2^{\circ}\right.$ e $3^{\circ}$ anos) do Ensino Fundamental. Brasília, DF.

Ministério da Educação. (2017). Base Nacional Comum Curricular. Educação é a base. Brasília, DF.

Nacarato, A. M., \& Custódio, I. A. (2018). O desenvolvimento do pensamento algébrico na educação básica: compartilhando propostas de sala de aula com o professor que ensina (ensinará) matemática. Brasília: Sociedade Brasileira de Educação Matemática. 
Oliveira, M. K. (1997). Vygotski, aprendizado e desenvolvimento: um processo sócio-bistórico. São Paulo: Scipione.

Pinto, G. U. (2010). Imaginação e formação de conceitos escolares: examinando processos dialógicos na sala de aula. (Tese de Doutorado). Universidade Metodista de Piracicaba, Piracicaba.

Prado, G.V.T. et al. (2011, setembro/dezembro). Narrativa pedagógica e memoriais de formação: escrita dos profissionais da educação? Revista Teias, 12(26), 143-153.

Radford, L. (2008). Iconicity and contraction: A semiotic investigation of forms of algebraic generalizations of patterns in different contexts. ZDM - The International Journal on Mathematics Education, 40(1), 83-96.

Radford, L. (2012). On the development of early algebraic thinking. PNA, 6(4), 117-133.

Radford, L. (2013). En torno a tres problemas de la generalización. In L. Rico, M C. Cañadas, J. Gutiérrez, M. Molina \& I. Segovia (Eds.). Investigación en Didáctica de la Matemática: homenaje a Encarnación Castro (pp. 3-12). Granada: Editorial Comares.

Radford, L. (2014). The progressive development of early embodied algebraic thinking. Mathematics Education Research Journal, 26, 257-277.

Ricoeur, P. (1989). Do texto à ação: ensaios de hermenêutica II. Porto: Rés.

Squalli, H. (2000) Une reconceptualisation du curriculum d'algèbre dans l'éducaction de base. Québec: Faculté des Sciences de l'Éduction, Université Laval.

Vale, I. (2006). Resolução de tarefas com padrões em contextos figurativos: exemplos de sala de aula. In I. Vale, I. Cabrita \& T. Cardoso. Matemática e Padróes no ensino básico: perspectivas e experiências curriculares de alunos e professores. Viana do Castelo: ESEVC - Projeto Padrões.

Van de Walle, J. (2009). Matemática no ensino fundamental: formação de professores e aplicação em sala de aula. Porto Alegre: Artmed.

Vygotski, L. S. (2007). A formação social da mente. São Paulo: Martins Fontes.

Vygotski, L. S. (2009). A construção do pensamento e da linguagem. São Paulo: Martins Fontes. 


\section{Notas de traducción}

i La educación escolar brasileña se divide en Educação Infantil (opcional) para niños menores de cinco años, Ensino Fundamental para niños entre 6 y 15 años y que comprende dos fases: Ensino Fundamental $I\left(1^{\circ}-5^{\circ}\right.$ año) y Ensino Fundamental II $\left(6^{\circ}-9^{\circ}\right)$, Ensino Médio (que dura 3 años), Ensino Técnico y Ensino Superior. La investigación descrita en este trabajo se realizó con alumnos del primer año de Ensino Fundamental, término que fue traducido como Educación Básica.

ii En portugués, las palabras "datos" (Definición RAE: "información sobre algo concreto que permite su conocimiento exacto o sirve para deducir las consecuencias derivadas de un hecho") y "dados" (Definición RAE: En los juegos de azar, objeto generalmente cúbico en cuyas caras aparecen puntos que representan distintos números o figuras diferentes) se escriben igual: "dados". De ahí la interpretación de la niña al entender como "dados" la palabra "datos" que había sido utilizada en el enunciado de la tarea.

iii “incompletitude": Término no traducible 